\title{
Comparison of the Level of Adherence to Laboratory Quality Management System between Public and Private Secondary Health Facilities in Southern Nigeria
}

\author{
Emma Akpan', Surajudeen Abiola Abdulrahman ${ }^{2} \&$ Nne Pepple $^{3}$ \\ ${ }^{1}$ Department of Laboratory Services, Resilient and Sustainable Systems for Health Programs Group, Management \\ Sciences for Health, 8 Sakete Close, Off Kampala Street, Wuse II, Abuja, Nigeria \\ ${ }^{2}$ Health Education England, East of England, 2-4 Victoria House, Capital Park, Fulbourn, Cambridge CB21 5XB, \\ United Kingdom \\ ${ }^{3}$ Department of Biochemistry, Salem University, Lokoja, Nigeria \\ Correspondence: Dr Surajudeen Abdulrahman, Health Education England, East of England, 2-4 Victoria House, \\ Capital Park, Fulbourn, Cambridge CB21 5XB, United Kingdom Tel: 44-772-859-6059. E-mail: \\ abdulsuraj@gmail.com
}

Received: May 21, 2020 Accepted: September 24, 2020 Online Published: October 19, 2020

doi:10.5539/gjhs.v12n12p27 URL: https://doi.org/10.5539/gjhs.v12n12p27

\begin{abstract}
Background: To achieve improved health outcomes of HIV positive patients receiving ART services, quality laboratory services must form an essential part of the services provided. The aim of this study was to compare service quality by assessing the level of adherence to quality system essentials (QSEs) in laboratory services delivered by public and private health institutions in Southern Nigeria.

Methods: This was an analytical cross-sectional study conducted among 50 health facilities' laboratories in 5 Southern States (Akwa Ibom, Anambra, Cross River, Edo and Rivers) of Nigeria. Randomly selected sample of secondary health facilities' laboratories (ten per state, and a total of 25 public and 25 private health facilities) receiving equal level of support from the same USAID/PEPFAR implementing partner and had been providing ART services to clients for a minimum of one year, were included in the study. Quarterly Internal quality audit was conducted in the ART Laboratory section of the selected health facilities spanning July 2015 to September 2016. 200 audit reports were checked, cleaned, and analyzed using SPSS version 23. We analyzed changes in mean performance scores over time across 6 quality management essentials using Repeated Measures ANOVA. Results were considered significant at $\mathrm{P}<0.05$.
\end{abstract}

Results: The result of the study showed that the private health facilities laboratory achieved a significantly higher improvement in Facility and Safety score over time $(p=0.019)$ compared to public health facilities. Overall, temporal improvements were recorded in all facilities in three out of the six QSEs (document and record, $p=0.045$; organization and personnel, $\mathrm{p}=0.020$; equipment, $\mathrm{p}<0.001)$ and total laboratory quality score $(\mathrm{p}=0.004)$. But there was no significant quarterly difference in performances on QSEs between public and private health facilities laboratories.

Conclusion: Our findings indicate that despite receiving the same level of external support, private hospital laboratories adhere better to the quality standards on Facility and safety than public hospital laboratories in Southern Nigeria.

Keywords: adherence, laboratory quality management system, quality system essential, private, public

\section{Background}

Quality Management could be defined as activities coordinated to direct and control organizations with regards to quality (International Organization for standardization, 2012). The origin of quality management is dated back to the $20^{\text {th }}$ century. In 1920, a method for statistical process control was developed by Shewhart; this formed the basis for quality control in the laboratory (Rohde, 2014). According to WHO, laboratory quality management system (QMS) involves the organization of laboratory processes and procedures into an understandable and workable structure - a path of workflow - predicated upon a set of building blocks or coordinated activities otherwise 
described as quality system essentials (QSE) (World Health Organization [WHO], 2011). The QSEs include Organization, Personnel, Equipment, Purchasing and Inventory, Process control/management, Information Management, Documents and records, Non-conforming Event/Occurrence Management, Assessment, Continual/Process Improvement, Customer Focus/Service, and Facilities and Safety. Ensuring accuracy and reliability in managing the QSEs will invariably result in good QMS which will further translate into better service delivery and quality of care.

To achieve any health target, strong laboratory systems and network capable of providing high quality services are a critical component of the health system, and plays a key role in routine diagnosis, care, treatment, and disease surveillance (Carraro \& Plebani, 2007). Clinical laboratory services impact directly on several aspects of patient care, such as patients' safety, duration of stay in health facility, resources use and customer satisfaction, among others (Harrison \& McDowell, 2008). The clinical laboratory officers are responsible for provision of information and services that contribute maximally to the effective delivery of care in the healthcare system. To provide accurate information to that effect is to ensure that the right test is performed on the right person, at the right time, using the right samples, following the right algorithm and Standard Operating Procedure (SOP), and finally producing accurate test results that will guide the providers to make the right diagnostic and therapeutic decisions.

The Nigerian Health system is relatively poorly organized and health care programs and services are poorly managed (Welcome, 2011). Nigeria as a country is still at the stage of responding to the HIV/AIDS epidemic and therefore lacks basic infrastructure on ground to render a lasting quality solution to health care challenges posed by both infectious and non-infectious diseases. Furthermore, clinical laboratories are grossly inadequate with weak infrastructure (Abimiku, 2009), and the level of implementation of Quality Management Systems (QMS) is deplorable, unlike in other developing countries (Okwuraiwe et al., 2012; Guindo et al., 2012).

According to a 2011 study by Polsa and colleagues (Polsa, Spens, Soneve, \& Antai, 2011), great disparity exists between the quality of healthcare services provided by the private and public health sectors in Nigeria. A section of the population believe that the private sector is usually more expensive as they offer a higher quality of services that are also more sustainable than the public sector in which services have been subsidized by Government. The public sector on the other hand is believed to have more qualified professionals in the health sector, but there is usually lapses in terms of adherence to standards, which compromises the service quality. Management styles also vary across the two sectors (public and private). While the private sector is flexible and easily adapt to changes, the public sector is somewhat rigid, having issues with programs of change and stifled by bureaucracy. Staff in the private sector are also perceived to be more committed and dedicated to their work, more caring and patient-focused than those in the public sector. While the foregoing reflects the general perception among Nigerians, research evidence remained inconclusive in this regard (Basu, Andrews, Kishore, Panjabi \& Stuckler, 2012), particularly because studies in this area have been very sparse in developing countries (Huseyin, Erdogan \& Katircioglu, 2008). This study examined laboratory QSEs and their implementation level between the two institutions, with an overall aim of comparing service quality by assessing the level of adherence to quality management system in laboratory services delivered by public and private health institutions in Southern Nigeria.

\section{Methods}

\subsection{Study Design, Population and Location}

This was an analytical cross-sectional study conducted across five States of the Southern (South - South and SouthEast) region of Nigeria, where the burden of HIV/AIDS is highest. These states are: Akwa Ibom, Anambra, Cross River, Edo and Rivers. The study sample was secondary health facilities' laboratories from the five states mentioned above. Fifty facilities that met the inclusion criteria were randomly selected for the study. Selected facilities received equal level of support (source of funding and technical assistance being mainly from PEPFAR through FHI 360) and have been providing ART services to clients for a minimum of one year (i.e inclusion criteria).

\subsection{Sample Size and Sampling Procedure}

To detect a difference of at least $20 \%$ in Facility and Safety score between private and public health facilities, we determined using formula for calculating sample size in hypothesis testing by comparing two proportions (Lemeshow, Hosmer, Klar, Lwanga, \& World Health Organization, 1990), that 46 health facilities would provide at least $80 \%$ power assuming a two-tailed test and type 1 error rate of $5 \%$. We increased the sample size to 50 to account for approximately $10 \%$ non-response. Ten (10) secondary health facilities ( 5 private and 5 public) that met the inclusion criteria were randomly selected from each of the 5 selected States of the Southern region of Nigeria, using a simple random sampling technique (lottery method). Table 1 provides a summary of the characteristics of 
the 50 facilities that were audited and analyzed in this study.

\subsection{Ethical Approval and Consent to Participate}

An ethical exemption was obtained for this study according to the National Code of Health Research Ethics, Section B, Clause "G" of Exemption from the Ministry of Health of the various States. A signed informed consent was obtained from the respective head of laboratories of the institutions where the studies were conducted before commencement of data collection, to enable the researchers a full consent to use their data.

\subsection{Data Collection}

Data was collected using an internal audit tool/checklist which was a simple extract from the standard assessment tool from World Health Organization/ Africa Regional Office Stepwise Laboratory Improvement Process towards Accreditation (WHO/AFRO SLIPTA) (World Health Organization [WHO], 2010). Although the comprehensive tool has 12 sections based on the 12 known laboratory quality system essentials (ISO, 2015); the internal audit tool, designed from 6 of these 12 sections (Assessment, Organization, Personnel, Equipment, Document and record, Facilities and Safety) was routinely used to conduct internal quality audit for the selected health facilities' laboratories by FHI360 laboratory technical officers, facility heads of laboratory departments and laboratory focal persons from government agencies/departments vested with oversight responsibilities. This tool allowed for simplicity and easy self-administration on a regular basis by these facilities (Figure 1). The quality system essentials - with various percentage weighting - that were considered are: 1) Documents and records (12\%); 2$)$ Organization and personnel (11\%); 3) Internal and external quality assessment (30\%); 4) Inventory control system (13\%); 5) Equipment (13\%); and 7) Facility and safety (21\%). The modified assessment check list consists of a total of 46 questions with a response scale of "yes" "no" and "partial". Full points were awarded if all requirement were fulfilled, partial/half points when some requirements were not fulfilled, and no point when all requirements were not fulfilled. Marks were awarded based on direct observation, review of records and documents, direct open-ended question to staff or combination of all in some cases. Standardized guidelines on scoring were provided for reference and consistency. The researchers worked with the facilities' quality officers to conduct internal audit for 2 quarters of year 2016 (January - March, April - June 2016) while reports of 2 rounds of audit conducted between July to December 2015 were also collated and analyzed to make up four quarters (one year) audit report. Overall, there were four audit reports per facility and a total of 200 audit reports were analyzed at the end of the data collection.

The maximum achievable score was 12 for Documents and Records, 11 for Organization and Personnel, 30 for Internal and External Quality Assessment, 13 for Inventory Control and Equipment respectively, and 21 for Facility and Safety. Minimum score for all QSEs was 0. Higher scores denotes better performance and incremental/temporal improvements were expected with each quarterly audit. All facilities were encouraged and supported to implement continuous quality improvement (CQI) programs to address areas of poor performance in each audit cycle. The CQI programs implemented are variable and dependent on the specific area of poor performance recorded during quarterly audit in each hospital laboratory (see Figure 1), with specific activities targeted at shoring up performance towards meeting expected standards in each QSE domain (see supplementary file). 


\begin{tabular}{|c|c|c|c|c|c|}
\hline & \multicolumn{5}{|c|}{ INTERNAL LABORATORY QUALITY AUDIT TOOL } \\
\hline & \multicolumn{5}{|l|}{ Facility Name: } \\
\hline & \multicolumn{2}{|l|}{ Audited By: } & & & \\
\hline & \multirow{2}{*}{\multicolumn{5}{|c|}{\begin{tabular}{|lll} 
Reviewed By: & Signature: & Date : \\
explanation or further comments for each "partial" or "no" response. Award scores appropriately as prov
\end{tabular}}} \\
\hline & & & & & \\
\hline & QUALITY ESSENTIALS ASSESSED & $\mathbf{Y} / \mathbf{N}$ & $\mathbf{P}$ & POINTS & SCORE \\
\hline \multirow[t]{8}{*}{1.0} & Documents \& Records & & & & \\
\hline & - Are Lab registers and worksheets available and up to date? & & & 3 & \\
\hline & - Is there an up to date record of Lab monthly Summary forms ? & & & 1 & \\
\hline & - Is there an up to date record of CRRIF ? & & & 2 & \\
\hline & - Are SOPs, Manuals and Job Aids available, current and easily accessible & & & 3 & \\
\hline & - Are SOPs read and signed by relevant personnels ? & & & 1 & \\
\hline & $\begin{array}{l}\text { - Are the personnel familiar with relevant SOPs? (Assess staff competency; ask } \\
\text { questions!) }\end{array}$ & & & 2 & \\
\hline & Documents & Recor & total & 12 & \\
\hline \multirow[t]{8}{*}{2.0} & Organization \& Management & & & & \\
\hline & - Is there an organizational Chart? & & & 1 & \\
\hline & - Does organizational chart describe heirachical personnel relationship in the laboratory? & & & 1 & \\
\hline & $\begin{array}{l}\text { Are there personnels files? Do they contain copies of staff qualifications and practicing } \\
\text { licence where applicable? }\end{array}$ & & & 3 & \\
\hline & - Are job descriptions available in the personnel files ? & & & 2 & \\
\hline & - Is there evidence of technical supervision ? e.g. review of test results/EQA/IQC reports? & & & 3 & \\
\hline & - Is there evidence of training and retraining of staff? & & & 1 & \\
\hline & Organization d & ersonn & total & 11 & \\
\hline 3.0 & Internal \& External Quality Assess ment & & & & \\
\hline & - Is there an SOP available for running IQC including frequency of runs ? & & & 3 & \\
\hline & - Is there an up to date IQC records for all the laboratory assays ? & & & 5 & \\
\hline & - Are charts (LJ) being used to document / display IQC results ? & & & 3 & \\
\hline & - Is the laboratory registered for EQA - PT / ILC for the tests performed ? & & & 2 & \\
\hline & - Are PT / ILC records up to date and well arranged for all the registered assays ? & & & 5 & \\
\hline & - Did the laboratory participate in the most recent two PT trials ? & & & 2 & \\
\hline & - Has the lab achieved at least $80 \%$ on the most recent two CD4 PT surveys ? & & & 5 & \\
\hline & - Are there records of CAPA documentation for the poor IQC/EQA/ internal audits? & & & 2 & \\
\hline & - Are internal audits conducted by the Quality Officer \& reviewed by HOD? & & & 3 & \\
\hline & Internal \& External Quality & sessme & total & 30 & \\
\hline 4.0 & \begin{tabular}{|l|} 
Inventory Control System \\
\end{tabular} & & & & \\
\hline & - Has the laboratory provided uninterrupted services in the last one month? & & & 2 & \\
\hline & - Is the store clean,well arranged, monitored (temperature) and free from dust \& pests ? & & & 2 & \\
\hline & - Are tally card available for all inventory items with up to date records ? & & & 2 & \\
\hline & - Are CRIFF records available and completed for the last two reporting period? & & & 5 & \\
\hline & - Are reagents and consumables within manufacturer's assigned expiry dates ? & & & 2 & \\
\hline & \begin{tabular}{|ll} 
& Inventory Con
\end{tabular} & l Syste & total & 13 & \\
\hline & 5.0 Equipment & & & & \\
\hline & - Is equipment maintenance log (service / repair) available \& up to date for ALL equipment? & & & 2 & \\
\hline & - Are records of service reports available and up to date for each equipment? & & & 2 & \\
\hline & - Is routine preventive maintenance by operators performed \& documented on the chart? & & & 5 & \\
\hline & - Are Air Condition (AC) units adequate and functional ? & & & 2 & \\
\hline & - Are installation / validation records available for each equipment ? & & & 1 & \\
\hline & - Are all major and ancillary equipment covered when not in use to prevent dust ? & & & 1 & \\
\hline & & & & 2 & \\
\hline & & uipme & total & 13 & \\
\hline & 6.0 Facility \& Safety & & & & \\
\hline & - Is there an adequate electricity with back-up generator and stabilisers/UPS ? & & & 2 & \\
\hline & - Are there appropriate leak proof,colour coded bins with liners for lab waste? & & & 2 & \\
\hline & - Are wastes segregated into general, infectious and sharps ? & & & 2 & \\
\hline & - Are PEP guidline \& lab signs (caution \& biohazard signs etc) available and enforced? & & & 2 & \\
\hline & - Are sockets safe \& functional, wires properly located and protected from traffic? & & & 2 & \\
\hline & - Are valid fire extinguishers and other gadgets available? Are staff trained on their use? & & & 2 & \\
\hline & - Is the floor clean and tidy and cleanings documented ? & & & 2 & \\
\hline & - Is there a clean area in the lab for record update and other purposes? & & & 1 & \\
\hline & - Is there a first aid box with appropriate content and in the right location? & & & 1 & \\
\hline & - Are PPEs: Laboratory Coat, Handgloves, eye goggle available and consistently used ? & & & 3 & \\
\hline & - is there eye wash \& hand wash staions with required solutions / materials? & & & 2 & \\
\hline & e & d Safe & b total & 21 & \\
\hline & BONUS FOR OBSERVED GOOD LAB PRACTICES (GLP) & & & 5 & \\
\hline & TOTAL & & & 100 & \\
\hline & SUMMARY OF AUDIT SCORES & & & MAX & SCORE \\
\hline & 1.0 Documents \& Records & & & 12 & $\mathbf{0}$ \\
\hline & \begin{tabular}{|l|} 
2.0 Organization \& Personnel \\
\end{tabular} & & & 11 & $\mathbf{0}$ \\
\hline & 3.0 Internal \& External Quality Ass essment & & & 30 & $\mathbf{0}$ \\
\hline & 4.0 Inventory Control System & & & 13 & $\mathbf{0}$ \\
\hline & 5.0 Equipment & & & 13 & $\mathbf{0}$ \\
\hline & 6.0 Laboratory Signs/Bench Top References & & & 17 & \\
\hline & 6.0 Facility \& Safety & & & 21 & $\mathbf{0}$ \\
\hline & TOTAL SCORES & & & 100 & $\mathbf{0}$ \\
\hline
\end{tabular}

Figure 1. Internal Audit Checklist/Scoring Tool 

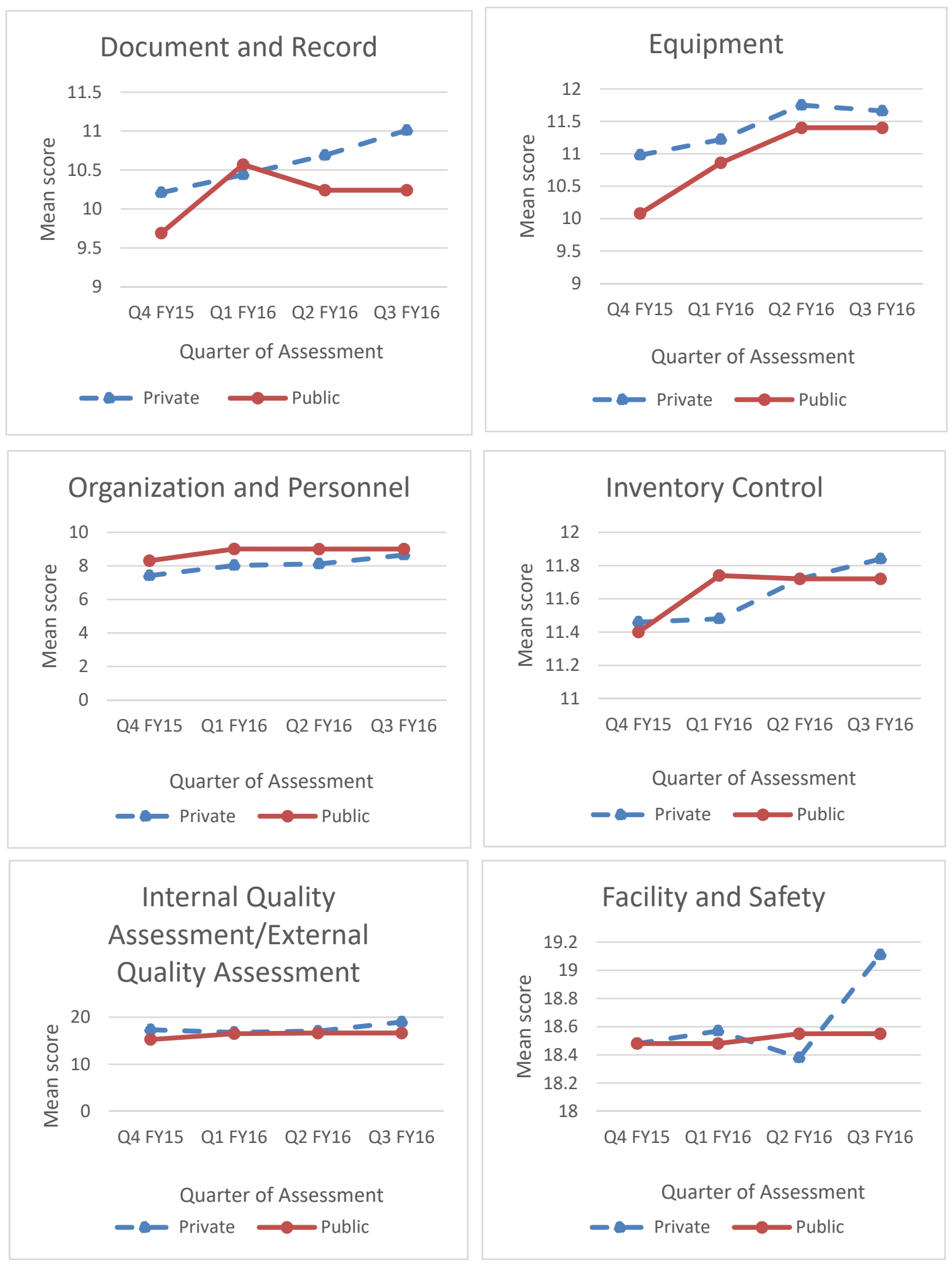

Figure 2. Graph of 2-way repeated measures ANOVA showing changes in QSE parameters by time, group and group $\mathrm{x}$ time interaction effect $(\mathrm{n}=50)$ 
Table 1. Characteristics of secondary health facilities involved in the study $(n=50)$

\begin{tabular}{|c|c|c|c|c|c|c|c|c|c|c|}
\hline \multirow{2}{*}{$\mathrm{S} / \mathrm{n}$} & \multirow{2}{*}{ Facility } & \multirow{2}{*}{ States } & \multicolumn{2}{|c|}{ Coordinate } & \multirow[b]{2}{*}{ Affiliation } & \multirow{2}{*}{ Location } & \multirow{2}{*}{$\begin{array}{l}\text { Current number } \\
\text { receiving ART as at } \\
\text { May 2017 }\end{array}$} & \multirow{2}{*}{$\begin{array}{l}\text { Date } \\
\text { commencement } \\
\text { ART }\end{array}$} & \multirow{2}{*}{$\begin{array}{l}\text { Laboratory } \\
\text { Set-up }\end{array}$} & \multirow{2}{*}{$\begin{array}{c}\text { No of lab } \\
\text { personnel* }^{*}\end{array}$} \\
\hline & & & Latitude & Longitude & & & & & & \\
\hline 1 & Ekpene Obom QIC Rehabilitation Hospital & Akwa-Ibom & 4.8619 & 7.8205 & Private & Rural & 583 & 1-Oct-2013 & ART & 4 \\
\hline 2 & Etim Ekpo Divine Love Hospital & Akwa-Ibom & 5.005163 & 7.6227 & Private & Rural & 129 & 1-Aug-2013 & ART & 2 \\
\hline 3 & Ituk Mbang Methodist General Hospital & Akwa-Ibom & 4.9172 & 8.0273 & Private & Rural & 1,466 & 1-Oct-2013 & ART & 3 \\
\hline 4 & Mercy Hospital & Akwa-Ibom & 4.9847 & 7.7909 & Private & Rural & 554 & 1-Oct-2013 & ART & 6 \\
\hline 5 & Uyo Gateway Hospital Uyo & Akwa-Ibom & 5.3956 & 7.7738 & Private & Urban & 27 & 1-Apr-2014 & ART & 2 \\
\hline 6 & Etim Ekpo General Hospital & Akwa-Ibom & 4.9938 & 7.6050 & Public & Rural & 1,161 & 1-Oct-2013 & ART & 3 \\
\hline 7 & Etinan General Hospital & Akwa-Ibom & 4.8263 & 7.8661 & Public & Urban & 1,348 & 1-Oct-2013 & ART & 6 \\
\hline 8 & Ikono General Hospital & Akwa-Ibom & 5.2827 & 7.7131 & Public & Rural & 213 & $1-O c t-2013$ & ART & 2 \\
\hline 9 & Ikot Ekpene General Hospital & Akwa-Ibom & 5.1747 & 7.7133 & Public & Urban & 2,713 & 1-Oct-2013 & ART & 4 \\
\hline 10 & Oron General Hospital (Iquita) & Akwa-Ibom & 4.8190 & 8.2340 & Public & Urban & 4,173 & 1-Jan-2008 & ART & 6 \\
\hline 11 & Adazi St Joseph Hospital & Anambra & 6.1023 & 7.0304 & Private & Rural & 1,102 & 13-Apr-2013 & ART & 4 \\
\hline 12 & Holy Rosary Hospital & Anambra & 6.1599 & 6.7752 & Private & Urban & 1,037 & 13-Apr-2013 & ART & 12 \\
\hline 13 & Immaculate Heart Hospital (Nkpor) & Anambra & 6.3310 & 6.8916 & Private & Urban & 837 & 15-Nov-2005 & ART & 3 \\
\hline 14 & Our Lady of Lourdes Hospital & Anambra & 5.8629 & 6.8618 & Private & Urban & 2,260 & 13-Apr-2013 & ART & 4 \\
\hline 15 & St Charles Borromeo Hospital & Anambra & 6.1434 & 6.8181 & Private & Urban & 2,566 & 13-Jul-2005 & ART & 4 \\
\hline 16 & Ekwulobia General Hospital & Anambra & 6.0181 & 7.0759 & Public & Rural & 785 & 14-Mar-2007 & ART & 4 \\
\hline 17 & Iyi-Enu Hospital & Anambra & 6.1545 & 6.8497 & Public & Urban & 334 & 13-Mar-2013 & ART & 9 \\
\hline 18 & Neni Comprehensive Health Centre NAUTH & Anambra & 6.0749 & 6.9761 & Public & Rural & 762 & 13-Sep-2013 & ART & 6 \\
\hline 19 & Oba Comprehensive Health Centre (Trauma Centre) & Anambra & 6.0593 & 6.8332 & Public & Rural & 1,447 & 13-Sep-2013 & ART & 5 \\
\hline 20 & Ukpo Comprehensive Health Centre & Anambra & 6.1977 & 6.9707 & Public & Rural & 603 & 13-Sep-2013 & ART & 6 \\
\hline 21 & Akpabuyo St Joseph Hospital & Cross River & 8.4678 & 4.9158 & Private & Rural & 518 & 13-Oct-2013 & ART & 5 \\
\hline 22 & Holy Family Catholic Hospital & Cross River & 5.9553 & 8.7150 & Private & Urban & 1986 & 28-Jun-2005 & ART & 7 \\
\hline 23 & Sacred Heart Catholic Hospital, Obudu & Cross River & 6.6743 & 9.1700 & Private & Rural & 748 & $1-O c t-2013$ & ART & 8 \\
\hline 24 & Yala Lutheran Hospital & Cross River & 6.4694 & 8.4972 & Private & Rural & 213 & 13-May-2013 & ART & 5 \\
\hline
\end{tabular}




\begin{tabular}{|c|c|c|c|c|c|c|c|c|c|c|}
\hline 25 & Ogoja Catholic Maternity Hospital & Cross River & 6.6647 & 8.8268 & Private & Urban & 2,368 & 1-Oct-2013 & ART & 7 \\
\hline 26 & Akpet Central Cottage Hospital & Cross River & 5.6183 & 8.0964 & Public & Rural & 395 & 29-Jun-2005 & ART & 4 \\
\hline 27 & Okpoma General Hospital & Cross River & 6.6152 & 8.6697 & Public & Rural & 81 & 13-May-2013 & ART & 3 \\
\hline 28 & Obudu clinic & Cross River & 6.6560 & 9.1630 & Public & Rural & 96 & May, 2013 & ART & 3 \\
\hline 29 & Ogoja General Hospital & Cross River & 6.6521 & 8.7942 & Public & Urban & 2,607 & 1-Oct-2013 & ART & 10 \\
\hline 30 & Ugep General Hospital & Cross River & 5.8085 & 8.0723 & Public & Urban & 1,203 & 29-Jun-2005 & ART & 6 \\
\hline 31 & Faith Mediplex & Edo & 6.3153 & 5.6036 & Private & Urban & 1,192 & Oct. 2013 & ART & 11 \\
\hline 32 & Uromi Central Hospital & Edo & 6.7135 & 6.3283 & Private & Urban & 1,128 & 1-Jan-2008 & ART & 3 \\
\hline 33 & St. Camillus Hospital & Edo & 6.7135 & 6.3283 & Private & Urban & 792 & Oct. 2013 & ART & 4 \\
\hline 34 & Stella Obasanjo Hospital & Edo & 6.2741 & 5.6304 & Private & Urban & 473 & Oct. 2013 & ART & 10 \\
\hline 35 & Oben Cottage Hospital & Edo & 5.9901 & 5.8957 & Private & Urban & 66 & Feb. 2007 & ART & 3 \\
\hline 36 & Afuze General Hospital & Edo & 6.9734 & 6.0368 & Public & Rural & 25 & Oct. 2013 & ART & 2 \\
\hline 37 & Agenebode General Hospital & Edo & 7.1138 & 6.6666 & Public & Urban & 152 & April, 2013 & ART & 1 \\
\hline 38 & Auchi Central Hospital & Edo & 7.0728 & 6.2583 & Public & Urban & 2,280 & 1-Feb-2007 & ART & 4 \\
\hline 39 & Ubiaja General Hospital & Edo & 6.6411 & 6.3938 & Public & Urban & 83 & April, 2013 & ART & 1 \\
\hline 40 & Usen General Hospital & Edo & 6.7482 & 5.3457 & Public & Urban & 127 & April, 2013 & ART & 2 \\
\hline 41 & Pope John Paul II Hospital & Rivers & 4.6131 & 7.3941 & Private & Rural & 1,120 & 2-Jun-2012 & ART & 6 \\
\hline 42 & St Martins Hospital & Rivers & 6.1122 & 6.8016 & Private & Urban & 48 & 4-Oct-2013 & ART & 7 \\
\hline 43 & New Mile One Hospital & Rivers & 4.7908 & 6.9940 & Private & Urban & 90 & 3-Oct-2013 & ART & 4 \\
\hline 44 & Sonnabel Hospital & Rivers & 4.8282 & 6.9836 & Private & Urban & 34 & 13-Nov-2013 & ART & 4 \\
\hline 45 & Meridian Hospital & Rivers & 4.8009 & 6.9977 & Private & Urban & 86 & 15-Nov-2013 & ART & 6 \\
\hline 46 & Abua General Hospital & Rivers & 4.8545 & 6.6506 & Public & Rural & 113 & 8-Apr-2013 & ART & 2 \\
\hline 47 & Ahoada General Hospital & Rivers & 5.0961 & 6.6427 & Public & Urban & 3,443 & 3-Jun-2006 & ART & 5 \\
\hline 48 & Bonny General Hospital & Rivers & 4.4357 & 7.1750 & Public & Rural & 486 & 12-Dec-2012 & ART & 2 \\
\hline 49 & Degema General Hospital & Rivers & 4.7488 & 6.7645 & Public & Rural & 192 & 7-Apr-2013 & ART & 2 \\
\hline 50 & Isiokpo General Hospital & Rivers & 5.0080 & 6.8709 & Public & Urban & 390 & 23-Apr-2013 & ART & 3 \\
\hline
\end{tabular}

ART, Antiretroviral Therapy.

*Include at least one Laboratory Scientist, and reflects personnel status as at Q3 FY16. 
Table 2. Summary table of 2-way repeated measures ANOVA showing changes in QSE parameters by time, group and group $\mathrm{x}$ time interaction effect $(\mathrm{n}=50)$

\begin{tabular}{|c|c|c|c|c|c|c|c|}
\hline SOURCE & Q4 FY15 & Q1 FY16 & Q2 FY16 & Q3 FY16 & $\begin{array}{l}\text { "Group } \\
\text { main } \\
\text { effect" }\end{array}$ & $\begin{array}{l}\text { "Time } \\
\text { main } \\
\text { effect" }\end{array}$ & $\begin{array}{l}\text { "Group } \\
\text { X Time } \\
\text { interac } \\
\text { tion" }\end{array}$ \\
\hline \multicolumn{8}{|c|}{ Document \& record } \\
\hline Private & $10.21 \pm 0.12$ & $10.44 \pm 0.12$ & $10.69 \pm 0.09$ & $11.01 \pm 0.09$ & & & \\
\hline Public & $9.69 \pm 0.12$ & $10.57 \pm 0.12$ & $10.24 \pm 0.09$ & $10.24 \pm 0.09$ & 0.327 & $0.045^{*}$ & 0.277 \\
\hline \multicolumn{8}{|c|}{ Organization \& Personnel } \\
\hline Private & $7.42 \pm 0.14$ & $8.03 \pm 0.13$ & $8.12 \pm 0.13$ & $8.65 \pm 0.13$ & & & \\
\hline Public & $8.31 \pm 0.14$ & $9.01 \pm 0.13$ & $9.00 \pm 0.13$ & $9.00 \pm 0.13$ & 0.208 & $0.020 *$ & 0.705 \\
\hline \multicolumn{8}{|l|}{ IQA \& EQA } \\
\hline Private & $17.34 \pm 1.65$ & $16.78 \pm 1.59$ & $17.04 \pm 1.72$ & $19.00 \pm 1.60$ & & & \\
\hline Public & $15.26 \pm 1.65$ & $16.50 \pm 1.59$ & $16.62 \pm 1.72$ & $16.62 \pm 1.60$ & 0.554 & 0.156 & 0.281 \\
\hline \multicolumn{8}{|c|}{ Inventory Control } \\
\hline Private & $11.46 \pm 0.26$ & $11.48 \pm 0.24$ & $11.72 \pm 0.25$ & $11.84 \pm 0.23$ & & & \\
\hline Public & $11.4 \pm 0.26$ & $11.74 \pm 0.24$ & $11.72 \pm 0.25$ & $11.72 \pm 0.26$ & 0.945 & 0.111 & 0.593 \\
\hline \multicolumn{8}{|l|}{ Equipment } \\
\hline Private & $10.98 \pm 0.15$ & $11.22 \pm 0.15$ & $11.75 \pm 0.13$ & $11.66 \pm 0.12$ & & & \\
\hline Public & $10.08 \pm 0.15$ & $10.86 \pm 0.15$ & $11.40 \pm 0.13$ & $11.40 \pm 0.12$ & 0.214 & $0.001 *$ & 0.594 \\
\hline \multicolumn{8}{|l|}{ Facility \& Safety } \\
\hline Private & $18.48 \pm 0.12$ & $18.57 \pm 0.12$ & $18.38 \pm 0.12$ & $19.11 \pm 0.11$ & & & \\
\hline Public & $18.48 \pm 0.12$ & $18.48 \pm 0.12$ & $18.55 \pm 0.12$ & $18.55 \pm 0.11$ & 0.411 & 0.074 & $0.019^{*}$ \\
\hline \multicolumn{8}{|c|}{ Overall Lab Quality } \\
\hline Private & $74.52 \pm 3.40$ & $74.68 \pm 2.85$ & $76.26 \pm 2.92$ & $80.68 \pm 2.78$ & & & \\
\hline Public & $70.50 \pm 3.40$ & $75.82 \pm 2.85$ & $76.22 \pm 2.92$ & $76.22 \pm 2.78$ & 0.633 & $0.004 *$ & 0.161 \\
\hline
\end{tabular}

Values are Mean \pm Standard error; *Significant at $\mathrm{p}<0.05$; Group $\mathrm{x}$ time interaction represents difference in change in QSE scores from Q4 FY15 to Q3 FY16 between public and private facilities.

Table 3. Summary table of post-hoc analysis of Group X Time comparison pairs

\begin{tabular}{|c|c|c|c|c|c|c|}
\hline \multirow[b]{2}{*}{ Group-time comparison pairs } & \multicolumn{3}{|c|}{ Private $(n=25)$} & \multicolumn{3}{|c|}{ Public $(n=25)$} \\
\hline & $\begin{array}{l}\text { Mean } \\
\text { difference }\end{array}$ & $\begin{array}{l}95 \% \text { CI of } \\
\text { mean } \\
\text { difference }\end{array}$ & P value & $\begin{array}{l}\text { Mean } \\
\text { difference }\end{array}$ & $\begin{array}{l}95 \% \quad \text { CI of } \\
\text { mean } \\
\text { difference }\end{array}$ & P value \\
\hline \multicolumn{7}{|l|}{ Document \& Record } \\
\hline $\begin{array}{l}\text { Pair } 1 \\
\text { Q4 FY } 15 \text { vs Q1 FY } 16\end{array}$ & -0.23 & $-0.29--0.16$ & $<0.0001^{*}$ & -0.88 & $-0.95--0.81$ & $<0.0001 *$ \\
\hline $\begin{array}{l}\text { Pair } 2 \\
\text { Q4 FY } 15 \text { vs Q2 FY } 16\end{array}$ & -0.48 & $-0.54--0.42$ & $<0.0001^{*}$ & -0.55 & $-0.61--0.49$ & $<0.0001 *$ \\
\hline $\begin{array}{l}\text { Pair } 3 \\
\text { Q4 FY15 vs Q3 FY } 16\end{array}$ & -0.80 & $-0.86--0.74$ & $<0.0001^{*}$ & -0.55 & $-0.61--0.49$ & $<0.0001 *$ \\
\hline Pair 4 & -0.25 & $-0.31--0.19$ & $<0.0001^{*}$ & 0.33 & $0.27-0.39$ & $<0.0001 *$ \\
\hline
\end{tabular}


Q1 FY 16 vs Q2 FY 16

Pair 5

Q1 FY 16 vs Q3 FY 16

$-0.57 \quad-0.63--0.51 \quad<0.0001 * \quad 0.33 \quad 0.27-0.39 \quad<0.0001 *$

Pair 6

Q2 FY 16 vs Q3 FY 16

$\begin{array}{llllll}-0.32 & -0.37--0.27 & <0.0001 * & 0 & -0.05-0.05 & 1.000\end{array}$

Organization and Personnel

Pair 1

Q4 FY 15 vs Q1 FY 16

$\begin{array}{lllll}-0.61<0.69--0.53<0.0001 * & -0.70 \quad-0.78--0.62<0.0001 *\end{array}$

Pair 2

Q4 FY 15 vs Q2 FY 16

$\begin{array}{lllll}-0.70 & -0.78--0.62<0.0001 * & -0.69 & -0.77--0.61 \quad<0.0001 *\end{array}$

Pair 3

Q4 FY15 vs Q3 FY 16

$-1.23 \quad-1.31--1.15<0.0001^{*} \quad-0.69 \quad-0.77--0.61<0.0001^{*}$

Pair 4

Q1 FY 16 vs Q2 FY 16

$\begin{array}{llllll}-0.09 & -0.16--0.02 & 0.018 & 0.01 & -0.06-0.08 & 0.787\end{array}$

Pair 5

Q1 FY 16 vs Q3 FY 16

$\begin{array}{llllll}-0.62 & -0.69--0.55 & <0.0001 * & 0.01 & -0.06-0.08 & 0.787\end{array}$

Pair 6

Q2 FY 16 vs Q3 FY 16

$\begin{array}{llllll}-0.53 & -0.60--0.46 & <0.0001 * & 0 & -0.07-0.07 & 1.000\end{array}$

\section{Equipment}

Pair 1

Q4 FY 15 vs Q1 FY 16

$-0.33--0.15<0.0001^{*} \quad-0.78$

$-0.87--0.69$

$<0.0001 *$

Pair 2

Q4 FY 15 vs Q2 FY 16

$$
-0.77
$$

$-0.85--0.69$

$<0.0001 * \quad-1.32$

$-1.39--1.24$

$<0.0001^{*}$

Pair 3

Q4 FY15 vs Q3 FY 16

$-0.68$

$-0.76--0.60<0.0001^{*} \quad-1.32$

$-1.39--1.24$

$<0.0001 *$

Pair 4

Q1 FY 16 vs Q2 FY 16

$-0.53$

$-0.60--0.45<0.0001 * \quad-0.54$

$-0.62--0.46$

$<0.0001^{*}$

Pair 5

Q1 FY 16 vs Q3 FY 16

$-0.44$

$-0.52--0.36<0.0001 * \quad-0.54$

$-0.62--0.46$

$<0.0001 *$

Pair 6

Q2 FY 16 vs Q3 FY 16

0.09

$0.02-0.16$

0.014

$-0.07-0.07$

1.000

\section{Facility \& Safety}

Pair 1

Q4 FY 15 vs Q1 FY 16

$-0.16--0.02 \quad 0.011$

0

$-0.07-0.07$

1.000

Pair 2

Q4 FY 15 vs Q2 FY 16
0.10
$0.03-0.17$
$0.005^{*} \quad-0.07$
$-0.14--0.001$
0.045

Pair 3

Q4 FY15 vs Q3 FY 16

$-0.69--0.56<0.0001^{*} \quad-0.07$

$-0.14--0.005$

0.037

Pair 4

Q1 FY 16 vs Q2 FY 16

0.19

$0.12-0.26<0.0001^{*} \quad-0.07$

$-0.14--0.001$

0.045

Pair 5

Q1 FY 16 vs Q3 FY 16

$\begin{array}{llllll}-0.54 & -0.61--0.47 & <0.0001 * & -0.07 & -0.14--0.005 & 0.037\end{array}$




\begin{tabular}{lllllll}
\hline Pair 6 & -0.73 & $-0.79--0.66$ & $<0.0001^{*}$ & 0 & $-0.07-0.07$ & 1.000 \\
Q2 FY 16 vs Q3 FY 16 & & & & & \\
\hline Overall Lab Quality & & & & \\
Pair 1 & -0.16 & $-1.94-1.62$ & 0.858 & -5.32 & $-7.10--3.54$ & $<0.0001^{*}$ \\
Q4 FY 15 vs Q1 FY 16 & & & & & & \\
Pair 2 & -1.74 & $-3.54-0.06$ & 0.058 & -5.72 & $-7.52--3.92$ & $<0.0001^{*}$ \\
Q4 FY 15 vs Q2 FY 16 & & & & & & $<0.0001^{*}$ \\
Pair 3 & -6.16 & $-7.93--4.39$ & $<0.0001^{*}$ & -5.72 & $-7.49--3.95$ & \\
Q4 FY15 vs Q3 FY 16 & & & & & & \\
Pair 4 & -1.58 & $-3.22-0.06$ & 0.058 & -0.40 & $-2.04-1.24$ & 0.626 \\
Q1 FY 16 vs Q2 FY 16 & & & & & & \\
Pair 5 & -6.00 & $-7.60--4.39$ & $<0.0001^{*}$ & -0.40 & $-2.00-1.20$ & 0.618 \\
Q1 FY 16 vs Q3 FY 16 & & & & & & \\
Pair 6 & -4.42 & $-6.04--2.79$ & $<0.0001^{*}$ & 0 & $-1.62-1.62$ & 1.000 \\
Q2 FY 16 vs Q3 FY 16 & & & & & & \\
\hline
\end{tabular}

*Significant at $\mathrm{p}<0.008$ (Bonferroni correction for multiple pairwise comparisons).

\subsection{Data Analysis}

Data collected were checked, cleaned, and analyzed using SPSS version 23. Exploratory data analysis was performed to determine if there were missing data or outliers, as well as to examine the overall distribution of the data (normality testing). We used Repeated Measures ANOVA to assess changes in QSEs between and within-groups over time. We report results of between and within facility ANOVA, whereby "Group" i.e. the Type of Facility - Public vs Private - is the between facility factor, and "Time" (the quarterly assessments) - the within facility factor. We also examined the "Group x Time" interaction which represents the difference in change-from-baseline between the two groups. We did not consider any covariates in our analysis as all facilities received the same level of support (funding, equipment, maintenance/repairs, consumables, and technical assistance) from PEPFAR through FHI360 and oversight from laboratory focal persons from the respective State Ministry of Health. At least 2 laboratory personnel, one of whom must be a Laboratory Scientist, were required to set up an ART laboratory in the supported facilities and requisite mandatory training was provided to all personnel. $P$ value less than 0.05 was considered significant for all analysis.

\section{Results}

In this study, there were four points/quarters in which the six QSEs were measured, therefore, Repeated Measures ANOVA analysis was performed to examine differences in change in each QSE (and overall Laboratory Quality scores) over time, between private and public health facilities. The outcome of the analysis is summarized in Table 2.

In all cases, the assumption of sphericity was violated, therefore interpretation of the tests was done using the Hyund-Feldt test. The assumption of equality of variance was met ( $\mathrm{p}>0.05)$.

\subsection{Document and Record}

Overall, the analysis revealed a significant "Time main effect" in Document $\&$ Record score across all facilities i.e difference in mean Document \& Record score over time $(\mathrm{F}=2.987, \mathrm{df}=2.419,116.130, \mathrm{p}=0.045)$. In contrast, there was no significant "Group main effect" i.e between public and private facilities $(\mathrm{F}(1,48)=0.980, \mathrm{p}=0.327)$ or "Group X Time interaction" ( $\mathrm{F}=1.305, \mathrm{df}=2.419,116.130, \mathrm{p}=0.277$ ) for Document \& Record scores (Table 2).

\subsection{Organization and Personnel}

Results in Table 2 also showed a significant "Time main effect" in Organization \& Personnel score across all facilities $(\mathrm{F}=3.555, \mathrm{df}=2.666,127.971, \mathrm{p}=0.020)$. In contrast, there was no significant "Group main effect" ( $\mathrm{F}(1$, $48)=1.628, \mathrm{p}=0.208)$ or "Group X Time interaction" $(\mathrm{F}=0.436, \mathrm{df}=2.666,127.971, \mathrm{p}=0.705)$ for Organization $\&$ Personnel scores. 


\subsection{IQA and EQA}

The analysis revealed no significant "Time main effect" $(\mathrm{F}=1.813 \mathrm{df}=2.577,123.691, \mathrm{p}=0.156)$, "Group main effect" $(\mathrm{F}(1,48)=0.355, \mathrm{p}=0.554)$ or "Group X Time interaction" $(\mathrm{F}=1.291, \mathrm{df}=2.577,123.691, \mathrm{p}=0.281)$ in IQA \& EQA scores. (Table 2).

\subsection{Inventory Control}

There was no significant "Time main effect" $(\mathrm{F}=2.078, \mathrm{df}=2.762,132.592, \mathrm{p}=0.111)$, "Group main effect" ( $\mathrm{F}(1$, $48)=0.005, \mathrm{p}=0.945)$ or "Group $\mathrm{X}$ Time interaction" $(\mathrm{F}=0.615, \mathrm{df}=2.762,132.592, \mathrm{p}=0.593)$ for Inventory control scores (Table 2).

\subsection{Equipment}

There was a statistically significant "Time main effect" in Equipment score across all facilities $(\mathrm{F}=6.380, \mathrm{df}=$ $2.306,110.665, \mathrm{p}=0.001)$. In contrast, there was no significant "Group main effect" $(\mathrm{F}(1,48)=1.589, \mathrm{p}=0.214)$ or "Group X Time interaction" effect $(\mathrm{F}=0.565, \mathrm{df}=2.306,110.665, \mathrm{p}=0.594)$ in mean equipment scores. (Table 2).

\subsection{Facilities and Safety}

The analysis showed a statistically significant "Group X Time interaction" effect in mean Facilities \& Safety score $(\mathrm{F}=3.608, \mathrm{df}=2.681,128.692, \mathrm{p}=0.019)$, although no significant "Group main effect" $(\mathrm{F}(1,48)=0.687, \mathrm{p}=$ $0.411)$ or "Time main effect" $(\mathrm{F}=2.447, \mathrm{df}=2.681,128.692, \mathrm{p}=0.074)$ was observed in Facility and Safety scores (Table 2).

\subsection{Overall Laboratory Quality}

In addition to comparing change in individual QSE over time within and between facilities, the six QSE scores were mathematically combined to obtain an overall Laboratory Quality score for each quarter. This composite variable was tested to see if there were any significant differences in mean Total Laboratory Quality score over time within and between facilities. The analysis showed statistically significant changes in the outcome variable (Total Lab Quality score) over time i.e "Time main effect" $(\mathrm{F}=5.653 \mathrm{df}=2.115,101.523, \mathrm{p}=0.004)$. In contrast, there was no significant "Group main effect" $(\mathrm{F}(1,48)=0.231, \mathrm{p}=0.633)$ or "Group X Time interaction" effect (F $=1.843, \mathrm{df}=2.115,101.523, \mathrm{p}=0.161)$ in mean Total Lab Quality score (Table 2). In numerical terms, the Total Laboratory Quality score for both private and public health facilities improved by 6 percentage points from baseline to study endpoint (Q4 FY15 to Q3 FY16).

A graphical depiction of these results, particularly the interaction effect of time and group is depicted in Figure 2.

Post-hoc analysis was conducted to examine the differences in group-time pairs in order to ascertain the specific time points where the differences in performance of the health facilities lies. We present in Table 3 comparative data of quarter-on-quarter performance of both private and public health facilities, adjusted for multiple pairwise comparisons (Bonferroni correction). Not only did the private health facilities laboratories show significant differences in quarterly performance in document and records, organizational and personnel, and facility and safety, the magnitude of the mean differences also appeared generally larger as compared to the public facilities (Table 3). The results also showed no significant difference in performance between Q2 FY16 and Q3 FY16 for any of the QSEs and Total Laboratory Quality score among public health facilities $(\mathrm{p}=1.000)$.

\section{Discussion}

\subsection{Summary of Main Findings}

The result of the study showed that the private health facilities laboratory achieved a significantly higher improvement in Facility and Safety score over time $(\mathrm{p}=0.019)$ compared to public health facilities. Overall, temporal improvements were recorded in all facilities in three out of the six QSEs (document and record, $\mathrm{p}=0.045$; organization and personnel, $\mathrm{p}=0.020$; equipment, $\mathrm{p}<0.001)$ and Total laboratory quality score $(\mathrm{p}=0.004)$, but there was no significant quarterly difference in performances on QSEs between public and private health facilities laboratories. This indicates that the private hospital laboratories adhere better to the quality standards on Facility and safety compared to public hospital laboratories.

\subsection{Current (Expected) Standards of ART Laboratory Practices in Nigeria}

Different quality management essentials have their implementation and monitoring requirements according to ISO 15189 (International Organization for standardization, 2012); however, this study did not find any significant difference between private and public health facilities laboratories in the implementation of any of the QSEs. This is no surprise because all that is required by international standard and best practice recommendation is provided to 
these facilities (as part of the technical support provided by FHI360 through PEPFAR) as clear, easily implementable steps as far as routine services are being provided by the laboratories. A quick overview of the requirements is detailed in the supplementary file (S1).

Although significant improvements have been achieved in Nigeria over the recent years, the number of medical laboratories in Sub-Saharan Africa that meet International Quality Standards remain less than desirable (Schroeder \& Amukele, 2014).

\subsection{Comparison with Existing Literature}

In this study, a comparatively higher improvement in performance with regards to 'Facilities and Safety' score was observed in private hospital laboratories. This underscore notable users' differences in both attitude to safe working (including adherence to safety standards) and facility management's responsibility of providing a safe and clean work environment. Although the laboratory processes should be the same in this regard, there are inherent characteristics or factors (such as personnel attitude, environmental factors) in private and public health facilities that could be different from each other and affect the implementation and documentation of safety standards including the outcome of laboratory processes. It is possible that the observed improvements in performance of private hospital laboratories over time may not be unrelated to improved awareness and risk perception (at individual level) as well as better enforcement of operational policies and procedures regarding occupational safety by the hospital management.

Our findings contrast with the findings of a 2014 study conducted among 6 public health facilities laboratories in Nigeria, in which the authors report a sustained high performance of the facilities on facility and safety (Mbah et al., 2014). Like the findings of our study, the Iranian study by Soghra and colleagues reported that private hospital laboratories showed a $12 \%$ higher implementation rate with regards to safety policies and procedures than public hospital laboratories. They observed that the major problem area of public laboratories lies with waste management and providing personnel protective equipment. Albeit with a different population, focus and design, a 2014 study conducted by Asamole-Osuocha and colleagues among 242 HIV Testing and Counseling (HTC) sites across 25 states and FCT in Nigeria, revealed no significant differences in performance regarding safety between the public and private health facilities (Asamole-Osuocha et al., 2014).

Specifically, in terms of equipment, only a few private health facilities in Nigeria can afford adequate approved platforms for their tests, and usually have fewer staff to manage their laboratories. Therefore, when a private laboratory is privileged to equipment and reagents' support by any partner, they tend to show more commitment to maintaining them. This attitude is very different in public hospital laboratories whose support come from the government and because NGOs and other implementing partners easily go into collaboration with government facilities and agencies, funding for hospital services are easily available (Elbireer et al., 2013). In this study, private hospital laboratories appeared to adhere better to the standard requirements for equipment maintenance than the public hospital laboratories, evidenced in their documentations, and as shown by marginal quarterly differences in their mean scores. Given the sparsity of literature evidence from Nigeria, the finding of our study is supported by an Iranian study conducted by Anjarani and colleagues (Anjarani et al., 2013), in which they reported that public laboratories showed poorer performance than private laboratories on safety and equipment. A 2014 study conducted by Jegede et al among 25 public health facilities laboratories in North-west Nigeria, showed that there was no statistically significant difference in equipment and facility and safety scores over time in the studied facilities (Jegede et al., 2014).

An overview of these two essentials show a limit to which development partners could drive quality management within the health facilities. The responsibility of these two depend largely on the facilities' staff than on the supporting partner. For example, after the safe work environment and equipment have been provided; equipment start-up, routine maintenance and adherence to safety rules lie hugely on the facilities' staff.

From the authors' experience of working with private and public health facilities, it had been observed that the private facilities embrace support and their personnel are always willing to learn and implement the requirement of ISO standard than the public facilities. Because services in private hospitals are not subsidized, they tend to be more inclined to quality standards in order to keep their clients. This is largely because their survival depends to a great extent on market incentives (income from clients) which drives them towards provision of more effective and efficient services (Andaleeb, 2000). Again, management commitment is also observed to be better in private than the public health facilities, resulting in lesser protocol/bureaucracy and prompt response to service needs. In addition, the private facilities staff are directly supervised and monitored by the Executive Director (Chief Medical Director) who in most cases happen to be the owner of the facility. Also, private facilities scarcely have support for their activities, so they tend to be more appreciative of any support provided to them and generally demonstrate 
better commitment to doing what is required in order to sustain the support. Intermittently, private facilities provide incentives to their staff which is a form of motivation; salaries are also paid on time, without which staff could leave without prior notice and this could lead to service disruption. On the other hand, public facilities could owe their staff wages for months and still expect them to report to work, which is very demotivating. Furthermore, private facilities do not often concede to service disruption because they depend on income from clients for their operations. They also stand a risk of being shut down by government in case of epidemic outbreak, contamination, or any major non-conformity to standards. On the other hand, the public facilities are funded and maintained by government, placing less demand for self-sustainability on such facilities and inadvertently poorer accountability from staff and managers. Resource management and allocation for procurement of any commodity is at central level and series of procedures must be followed to achieve this, whereas the private facilities have less procedures and easy/fast disbursement of fund for their activities.

While some earlier studies by Polsa et al and Soghra et al have shown that the predictors of choice of public or private hospitals include patient perceptions of service quality and key demographic characteristics, the findings of the current study also agree with this position. Our findings suggest that private hospital laboratories demonstrate better adherence and commitment to provision of quality services evidenced by overall higher Total Laboratory Quality Score. Be that as it may, the comparative performance of public and private health care delivery systems remains a subject of ongoing debate. A 2012 systematic review by Basu et al (Basu et al., 2012) did not support the claim that the private sector is usually more efficient, accountable, or medically effective than the public sector. The authors opined that although the public sector frequently appears to be lacking timeliness and hospitality towards their clients, there was no significant difference between the two. As suggested by previous studies (Manickam \& Ankanagari, 2015), we believe that training and re-training of laboratory staff and enforcement of standard operational policies and procedures in laboratory practices are necessary to achieve needed improvements in quality of services.

Overall, our findings demonstrate a small to moderate effect size (Cohen's d $0.2-0.5$ ) for temporal improvements in mean scores of the QSEs reported in this study.

\subsection{Strengths}

Despite extensive literature review, we did not find any study directly comparing the QMS or QSE performance between private and public secondary health facilities' ART laboratory anywhere in Nigeria. Therefore, to the best of our knowledge, this study is the first to carry out this comparison in Nigeria. This analysis and the information provided is particularly useful in guiding the delivery of effective, qualitative, and responsive patients' care. The longitudinal nature of the study provided a means of observing changes in trend over a period of one year, and a means of demonstrating whether observed changes are sustained or not. Most importantly, it has been observed that although staff in private hospitals generally receive lesser wages than those in public hospitals, there appear to be other motivational factors which leads to their improved commitment and adherence to standards. These strategies, if adopted by the public hospitals, could lead to improved service quality.

\subsection{Limitations}

This study has some important limitations. The internal audit tool (checklist) used for this study may not have been comprehensive enough to accommodate all the quality indicators; as the simple checklist tends to focus more on indicators that speak to the laboratory's status in terms of service availability and accessibility and less on those factors to which adherence will translate directly to quality service. The use of Stepwise Laboratory Quality Improvement Towards accreditation (SLIPTA) checklist would have provided a more comprehensive assessment of QSEs and quality indicators that would easily spell out the relationship between adherence to the essentials and quality laboratory services. The design of this study, although longitudinal in nature, did not provide any objective means of isolating the specific factors or interventions that might have led to the observed improved performances in facility and safety among the private health facilities' laboratories as compared to the public health facilities' laboratories.

\section{Conclusion}

Beyond the significantly higher improvement in Facility and Safety score over time observed among private hospital laboratories in this study, all facilities demonstrated temporal improvements in performance in three of the six QSEs (document and record, organization and personnel, equipment) and Total Laboratory Quality score over the study period. Reassuring as this may be, it further emphasizes the need for ongoing technical support and continuous quality improvement particularly in the area of IQA and EQA and inventory control, which appear to be demonstrably lacking in all facilities in this study We believe that continuous and sustainable laboratory quality 
improvement, capacity building, focused management, follow up visits, sustained mentorship, advocacy, and commitment among all stakeholders are vital to maintain and improve on these results.

\section{Acknowledgements}

The authors would like to thank USAID/PEPFAR Nigeria, FHI360, Ministries of Health of the participating states, management of the health facilities and laboratory personnel that contributed to the successful conduct of this study.

\section{Author Contributions}

Conceived and designed the experiment: EA, SAA; Performed the experiment: EA, SAA; Analysed the data: SAA, EA; Wrote the paper: EA, SAA, NP; Reviewed the manuscript: SAA, EA, NP. The authors sincerely appreciate all participating facilities in this study and also acknowledge everyone whose effort led to the successful completion of this research. The critical roles of Uche Okudo, Osasere Oogieva, Blessing Airiagbonu, Olubunmi Omolade, Elizabeth Okoi, Mary Andy Nkwocha and Isi Ayi towards obtaining the informed consent of the participating facilities as well as the ethical approval of this study is well acknowledged.

\section{Authors' Note}

The datasets generated and/or analyzed during this study are available in a public data repository upon acceptance of this paper.

\section{Funding}

The author(s) received no financial support for the research, authorship, and/or publication of this article.

\section{Disclaimer}

The opinions expressed in the paper are strictly those of the authors, and does not represent the official position of FHI360, the respective state governments or health facilities on the subject.

\section{Competing Interests Statement}

The author(s) declared no potential conflicts of interest with respect to the research, authorship, and/or publication of this article.

\section{References}

Abimiku, A. L. G. (2009). Building Laboratory Infrastructure to Support Scale-Up of HIV/AIDS Treatment, Care, and Prevention: In-Country Experience. American Journal of Clinical Pathology, 131(6), 875-886. https://doi.org/10.1309/AJCPELMG6GX6RQSM

Andaleeb, S. S. (2000). Public and Private Hospitals in Bangladesh: Service Quality and Predictors of Hospital Choice. Health policy and planning, 15(1), 95-102. https://doi.org/10.1093/heapol/15.1.95

Anjarani, S., Safadel, N., Dahim, P., Amini, R., Mahdavi, S., \& Samiee, S. M. (2013). Establishment of National Laboratory Standards in Public and Private Hospital Laboratories. Iranian journal of public health, 42(1), 96.

Asamole-Osuocha, C., Mbah, H., Cartier, S., Ojo, E., Badru, T., Ibrahim, M., Oka, I., Egwa, E., \& Chabikuli, O. (2014). External quality assessment: On-site Evaluation of HIV Testing and Counselling Sites in Nigeria. Journal of AIDS and HIV Research, 6(4), 97-103. https://doi.org/10.5897/JAHR2013.0282

Basu, S., Andrews, J., Kishore, S., Panjabi, R., \& Stuckler, D. (2012). Comparative Performance of Private and Public Healthcare Systems in Low-And Middle-Income Countries: A Systematic Review. PLoS medicine, 9(6), e1001244. https://doi.org/10.1371/journal.pmed.1001244

Carraro, P., \& Plebani, M. (2007). Errors in a Stat Laboratory: Types and Frequencies 10 Years Later. Clinical Chemistry, 53(7), 1338-1342. http://dx.doi.org/10.1373/clinchem.2007.088344

Elbireer, A. M., Jackson, J. B., Sendagire, H., Opio, A., Bagenda, D., \& Amukele, T. K. (2013). The good, the bad, and the unknown: quality of clinical laboratories in Kampala, Uganda. PLoS One, 8(5), e64661. http://dx.doi.org/10.1371/journal.pone.0064661

Guindo, M. A., Shott, J. P., Saye, R., Diakité, M. L., Sanogo, S., Dembele, M. B., ... \& Diallo, D. A. (2012). Promoting good clinical laboratory practices and laboratory accreditation to support clinical trials in sub-Saharan Africa. The American journal of tropical medicine and hygiene, 86(4), 573-579. http://dx.doi.org/10.4269/ajtmh.2012.11-0691

Harrison, J. P., \& McDowell, G. M. (2008). The role of laboratory information systems in healthcare quality improvement. Int J Health Care Qual Assur, 21(7), 679-691. https://doi.org/10.1108/09526860810910159 
Huseyin, A., Erdogan, H. E., \& Katircioglu, S. T. (2008). Gearing Service Quality into Public and Private Hospitals in Small Islands: Empirical Evidence from Cyprus. International Journal of Healthcare Quality Assurance, 21(1), 8-23 https://doi.org/10.1108/09526860810841129

International Organization for Standardization ISO 15189:2012: Medical Laboratories Requirements for Quality and Competence. International Organisation for Standardisation, Geneva. Retrieved from http://www.iso.org

ISO 9001:2015 INTERNAL AUDIT; http://isoconsultantpune.com/iso-90012015-internal-audit/

Jegede, F. E., Mbah, H. A., Yakubu, T. N., Adedokun, O., Negedu-Momoh, O. R., \& Torpey, K. (2014). Laboratory quality audit in 25 anti-retroviral therapy facilities in North West of Nigeria. Open Journal of Clinical Diagnostics, 4(04), 193. https://doi.org/10.4236/ojcd.2014.44028

Lemeshow, S., Hosmer, D. W., Klar, J., Lwanga, S. K., \& World Health Organization. (1990). Adequacy of sample size in health studies. Chichester: Wiley.

Manickam, T. S., \& Ankanagari, S. (2015). Evaluation of quality management systems implementation in medical diagnostic laboratories benchmarked for accreditation. Journal of Medical Laboratory and Diagnosis, 6(5), 27-35. https://doi.org/10.5897/JMLD2015.0104

Mbah, H., Ojo, E., Ameh, J., Musuluma, H., Negedu-Momoh, O. R., Jegede, F., ... \& Udah, D. (2014). Piloting laboratory quality system management in six health facilities in Nigeria. PloS one, 9(12), e116185. https://doi.org/10.1371/journal.pone.0116185

Okwuraiwe, A. P., Audu, R. A., Meshack, E., Onwuamah, C. K., Okoli, C. L., Aleshinloye, R. O., ... \& Olatunji, M. (2012). Experience of quality management system in a clinical laboratory in Nigeria: lessons from the field. African Journal of Laboratory Medicine, 1(1), 1-5. http://dx.doi.org/10.4102/ajlm.v1i1.18

Polsa, P., Spens, K., Soneye, A., \& Antai, I. (2011). Comparing the perceived quality of private and public health services in Nigeria. Journal of Management Policy and Practice, 12(7), 18-26.

Rohde, R. E. (2014). The hidden profession that saves lives. Elsevier Connect. Retrieved from $\mathrm{http}: / / \mathrm{www}$.elsevier.com/connect/the-hidden-profession-that-saves-lives

Schroeder, L. F., \& Amukele, T. (2014). Medical Laboratories in Sub-Saharan Africa That Meet International Quality Standards. American Journal of Clinical Pathology, 141(6), 791-795. http://dx.doi.org/10.1309/AJCPQ5KTKAGSSCFN

Welcome, M. O. (2011). The Nigerian health care system: Need for integrating adequate medical intelligence and surveillance systems. Journal of pharmacy \& bioallied sciences, $3(4), 470$. https://doi.org/10.4103/0975-7406.90100

World Health Organization (WHO). (2010). Guide for the Stepwise Laboratory Improvement Process towards Accreditation in the African Region (Checklist). WHO, Geneva, 1-60.

World Health Organization (WHO). (2011). Laboratory Quality Management System Handbook, 2011. Retrieved 27 January, 2020, from https://apps.who.int/iris/bitstream/handle/10665/44665/9789241548274_ eng.pdf;jsessionid $=22 \mathrm{CF} 1 \mathrm{C} 17430 \mathrm{EA} 9 \mathrm{AA} 8 \mathrm{~A} 0151 \mathrm{~A} 7 \mathrm{AC} 1 \mathrm{BA} 137$ ? sequence $=1$

\section{Supplementary file}

\section{Current (Expected) Standards of ART Laboratory Practices in Nigeria}

Document and record: The standard requires that all necessary documents be available and up to date in the laboratories. These include Standard Operating Procedures (SOP) and job aids, laboratory registers and worksheets, monthly summary forms and bimonthly Combined Request, Requisition, Issue and Receipt Form (CRRIRF) reports. These are national tools that are provided to all facilities providing ART services to clients.

These document and records are essential part of daily laboratory operations that provide a guide for each testing procedures and record keeping. Monthly summary forms are monthly summary of services provided by the laboratory and bimonthly CRRIRF are summary of reagents and consumable usage by the laboratory. These two are necessary for quantification and resupply of laboratory commodities without which the laboratory cannot operate. These are of utmost importance to both private and public facilities.

Organization and Personnel Management: The standard requires that every personnel have a file in the facility and that the facility have a hierarchal relationship with personnel which should be depicted in 
organizational chart. There should also be evidence of staff academic qualifications, supervision by a superior as well as training and retraining. These are common records kept by every establishment and it is a prerequisite requirement for site activation for ART services, which are further strengthened through routine training, onsite mentoring and supportive supervisions by the supporting implementing partners. Thus, all sites would be at the same level for this requirement.

Internal and External Quality Assessment: The standard requires that internal quality control be run for all assay and there should be available SOPs for these. Control figures should also be recorded daily with Levey Jennings's chart. Laboratories should be registered for Proficiency testing for all assays run and incidents should be reported and corrective and preventive actions implemented where necessary. This element is well supported at the same level by the implementing partner providing supports for these facilities. All sites were observed to be registered for proficiency testing and the partner's presence were strongly felt in the facilities in terms of routine onsite mentoring for good clinical laboratory practices.

Inventory control system: The standard requires that laboratories provide uninterrupted services. The laboratory reagents and consumables are expected to be kept under optimum temperature and within the manufacturers' expiry date with inventory records (stock control/ tally cards) maintained and up-to-date. With the partner's support, every facility has a good inventory control system in place.

Equipment management: The standard requires that installation/validation be done on every equipment and records be maintained for that, as well as daily and routine preventive maintenance and services. The equipment are also expected to be covered when not in use and the laboratory must have a functional air conditioner to maintain the optimal temperature. A verifiable evidence of all these, in form of documentation, must be kept by all laboratories.

Facility and Safety: It is the responsibility of each facility's management to provide a safe work environment, but the responsibility of every staff to adhere to safety standards. Adherence to safety depends on individuals' attitudes e.g risk perception, ability to communicate risk and response to incidents. The policy demands that there be proper waste segregation into non-infectious, infectious and highly infectious wastes; there should also be some well- segregated work areas (clean - for record keeping, and dirty - for analysis). The laboratory floor and workstations are also expected to be clean and free of clutter, and there should be proper layout of laboratory with functional sockets and no crossing wires; eye and hand wash stations, first aid box, Post Exposure Prophylaxis (PEP) guidelines and fire extinguishers, which all staff must be familiar with their use.

\section{Copyrights}

Copyright for this article is retained by the author(s), with first publication rights granted to the journal.

This is an open-access article distributed under the terms and conditions of the Creative Commons Attribution license (http://creativecommons.org/licenses/by/4.0/). 